\title{
Un sistema de gestión de pavimentos basado en nuevas tecnologías para países en vía de desarrollo
}

\author{
A Pavement Management System Based on New Technologies \\ for Developing Countries
}

\author{
Macea-Mercado Luis Fernando \\ Universidad del Norte, Colombia \\ Correo:Imacea@uninorte.edu.co \\ Morales Luis \\ Universidad de Córdoba, Colombia \\ Correo.morales8430@hotmail.com
}

\author{
Márquez-Díaz Luis Gabriel \\ Universidad Pedagógica y Tecnológica de Colombia \\ Escuela de Transporte y Vías \\ Correo:luis.marquez@uptc.edu.co
}

Información del artículo: recibido: febrero de 2015, aceptado: octubre de 2015

\section{Resumen}

El acelerado crecimiento de la ciencia y la tecnología ha propiciado el desarrollo de soluciones cada vez más acertadas a los diversos problemas que enfrenta la sociedad moderna. En los procesos de gestión de pavimentos es posible desarrollar tecnologías de apoyo orientadas hacia la valoración funcional de pavimentos. El presente artículo propone y discute la implementación de un sistema para países en vía de desarrollo, que está basado en nuevas tecnologías que facilitan la toma y análisis de información para los procesos de gestión de pavimentos, con tiempos y costos razonables. El sistema propuesto utiliza un vehículo equipado con elementos que permiten el registro y localización geográfica automática de daños existentes en los pavimentos, así como la generación de mapas de deterioro vial a través de plataformas WEB, que se pueden utilizar para justificar la toma de decisiones en torno a políticas de inversión en infraestructura vial.

\section{Descriptores:}

- gestión de pavimentos

- seguridad vial

- nuevas tecnologías

- sistemas de información geográfica

- países en vía de desarrollo 


\begin{abstract}
The rapid growth of science and technology has led to the development of increasingly accurate solutions to the various problems facing modern society. In pavement management processes, it is possible to develop enabling technologies for functional assessment of pavements. This article proposes and discusses the implementation of a system for developing countries, which is based on new technologies which facilitate the collection and analysis of information for pavement management processes with low cost and reasonable time duration. The proposed system uses a vehicle equipped with components that automate the registration and geographic location of pavement damages and generate maps of road deterioration through WEB platforms, which can be used to support policy decisions on road infrastructure investment.
\end{abstract}

\section{Keywords:}

- pavement management

- road safety

- new technologies

- geographic information system

- developing countries

\section{Introducción}

Mantener en buen estado la infraestructura vial es importante para evitar sobrecostos de operación en el transporte de personas y bienes. Un país que permita el deterioro de su infraestructura vial tendrá sobrecostos de operación vehicular en un rango que se puede ubicar entre 1 y $3 \%$ de su producto interno bruto nacional (Bull, 2003). Además, la funcionalidad que debe ofrecer la red de carreteras de un país es crucial para la seguridad y comodidad de los usuarios. De ahí la importancia que tiene la gestión de pavimentos, concepto que surgió a finales de los años setenta (Diaz, 2014) y que ha evolucionado de forma acelerada en los últimos años, combinando diferentes actividades para proveer y administrar pavimentos. El objetivo básico de la gestión de pavimentos ha utilizado información segura y consistente para desarrollar criterios de decisión, posibilitar alternativas realistas de inversión y contribuir a la eficiencia en la toma de las decisiones (Solminihac, 2001).

Normalmente es más fácil administrar de una forma adecuada pequeños tramos viales, pero en una red extensa de carreteras esta labor se puede ver comprometida, ya que el inventariado de daños sobre la red vial es un aspecto crítico (Díaz, 2014; Thenoux y Halles, 2011), donde es necesario automatizar ciertos procesos para hacer más eficiente la recopilación y gestión de información de campo. En caso de no existir un mecanismo de gestión apoyado en procesos objetivos de obtención de información, se correría el riesgo de formular planes de poco alcance, reduciendo su efectividad, especialmente en países en vía de desarrollo, donde los recursos son escasos.

Colombia ha realizado grandes esfuerzos en materia de gestión vial. Entre los años 2005 y 2009, el Ministerio de Transporte y el Instituto Nacional de Vías (INVIAS) desarrollaron el programa de corredores de mantenimiento integral, involucrando 11 corredores viales importantes que cubrían una longitud de 1,987 $\mathrm{km}$. Esta iniciativa, que tuvo un costo aproximado de 1.2 billones de pesos colombianos, requería el diseño de un plan de acción que involucraba el inventariado de los daños del pavimento y la medición de parámetros funcionales como el International Roughness Index (IRI), cada $100 \mathrm{~m}$, para generar matrices de intervención que permitían mejorar el índice de servicio del corredor. El país también empleó la estrategia de administradores viales, que mediante la ejecución de 79 contratos, permite la atención de $11840 \mathrm{~km}$ de vías (INVIAS, 2013).

La identificación de intervenciones bajo estos dos esquemas se basa en dos criterios específicos: el criterio visual, que consiste en la categorización del estado de la vía en bueno, regular o malo, de acuerdo con la percepción del administrador vial, y el criterio técnico, que se deriva de la aplicación del manual para la inspección de pavimentos flexibles del INVIAS o del Standard Practice for Roads and Parking Lots Pavement Condition Index Surveys de la American Society for Testing and Materials (ASTM), que identifica y mide fenómenos de deterioro vial tales como: baches, fisuras, deformaciones, desprendimientos, ahuellamiento o desgaste, tanto en la calzada como en las bermas para posteriormente determinar, a través de algunos formatos, parámetros como el índice de condición del pavimento (Echeverry, 2014; Bull, 2003; Díaz et al., 2014). El segundo criterio requiere un riguroso proceso de medición que consume tiempo y tiene varios inconvenientes como el riesgo de hacer mediciones en el camino, la variabilidad entre diferentes auscultadores, el tamaño inadecuado de las muestras y el alto costo de la inspección (Solminihac, 2001; Lopez, 2010).

La calidad de la información de campo dependerá entonces de la asignación y priorización de recursos para mantenimiento, así como de la confiabilidad de las 
decisiones que se tomen en materia de intervención (Díaz, 2013). En consecuencia, es vital el desarrollo de tecnologías que permitan estudiar, analizar y comprender el comportamiento de los elementos de la infraestructura vial que, como los pavimentos, determinan buena parte de los costos de usuarios y requieren la mayor cantidad de recursos para su construcción y mantenimiento (Solminihac, 2001).

Como se discute en este artículo, se han desarrollado varios sistemas que mejoran la eficiencia de los procesos de inspección e inventariado de daños en los pavimentos con fines de administración vial. Sin embargo, la gran mayoría de estos sistemas no están concebidos para apoyar labores de gestión a nivel de red, sino a un nivel más focalizado, como es el caso de pequeñas secciones de vía, debido comúnmente al tipo de deterioro, pavimento y nivel de detalle en el que se enfocan (sólo grietas), lo que complica la toma de decisiones a nivel estratégico. Además, como muchos de ellos tienen costos elevados, se dificulta su implementación en países en vía de desarrollo.

En este contexto, el presente artículo desarrolla un sistema de apoyo a la gestión de pavimentos, de fácil acceso para los países en vía de desarrollo, orientado a facilitar la valoración del estado de las vías a nivel de red. Dicho sistema se fundamenta en la fusión de conceptos de arquitectura de computadoras y de sistemas operativos, para generar automáticamente registros de los daños presentes en las vías, así como en la conformación de mapas de deterioro vial a través de plataformas WEB, donde se describen los puntos con requerimientos de intervención. La gestión de esta información de campo apoya la toma de decisiones en torno a políticas de inversión en infraestructura vial, facilita el registro de la información de campo y fortalece los diagnósticos objetivos de evaluación.

\section{Antecedentes}

La manifestación de deterioros sobre la carpeta asfáltica de los pavimentos afecta negativamente la seguridad de la circulación vehicular, la hace menos confortable e incrementa los costos de operación (Solminihac, 2001). Por tal razón, diversas organizaciones, entidades gubernamentales e investigadores han centrado su atención en la gestión de pavimentos.

Para el Banco Mundial (BM), una buena gestión de infraestructura es indispensable para priorizar adecuadamente la inversión vial en las regiones. El BM utiliza el programa HDM-4 (Highway Development and Management) como principal herramienta de modelado y evaluación del comportamiento del pavimento, tomando en consideración aspectos como la calidad de rodadura, los costos de usuarios, los costos de conservación, construcción, y el valor residual de la vía (Miquel et al., 2007; Kerali, 2000; Solminhac, et al., 2003).

Agencias como el IRAP (International Road Assessment Program), emplean una metodología para la gestión de la seguridad vial basada en la medición del riesgo asociado con el estado de las carreteras. IRAP utiliza un vehículo especial para obtener información que permite clasificar el riesgo, desarrollar planes de inversión y analizar el IRI en diferentes tramos uniformes de carreteras (IRAP, 2009). En el año 2012, el gobierno colombiano contrató los servicios del programa IRAP para hacer un diagnóstico de 11,500 km de carreteras de la red nacional (Corporación Fondo de Prevención Vial, 2013). El vehículo utilizado tomó imágenes cada 100 m, a partir de las cuales equipos de investigadores analizaron las variables que afectan la seguridad vial (Universidad de los Andes, 2013). En este caso, el volumen de información recopilada por el vehículo hizo complejo y dispendioso el proceso de análisis, ya que la captura de la información de campo no estaba sujeta estrictamente a los puntos con daños o deficiencias.

La recolección de datos es una de las tareas más valiosas y costosas dentro de los programas de gestión de pavimentos (Díaz, 2013). Cada dato requiere tiempo, esfuerzo y dinero para ser recopilado, almacenado y recuperado. Su utilización permite identificar las necesidades de mantenimiento y rehabilitación, al igual que establecer prioridades para asignar fondos (Bennett et al., 2006). Los sistemas automáticos para la evaluación vial utilizan diversos equipos para asegurar la confiabilidad y eficiencia en la evaluación de los pavimentos. Estos equipos miden la localización (ej. GPS), rugosidad (ej. perfiles de precisión laser y manual), propiedades mecánicas del pavimento (ej. deflectómetro de impacto, penetrómetro dinámico de cono, viga Benkelman), deterioro superficial (ej. cámaras fotográficas de línea y de láser), y otras características importantes de la infraestructura vial (Bennett et al., 2006).

Se puede destacar el sistema PASCO ROADRECON desarrollado por la empresa Pasco Corporation, que trabajó en el desarrollo de un vehículo de inspección vial desde los años 60, el cual utiliza una cámara fotográfica de $35 \mathrm{~mm}$, instalada en la parte delantera de una camioneta para obtener registros del estado del pavimento y medir la rugosidad. Las fotografías capturadas se toman por la noche, utilizando un sistema que controla la cantidad y ángulo de iluminación. El vehículo opera a velocidades de hasta $80 \mathrm{~km} / \mathrm{h}$ y fotografía un ancho de $5 \mathrm{~m}$. La evaluación del pavimento solo requiere interpretación visual de las fotografías (Solminihac, 2001). 
El sistema GERPHO almacena imágenes continuas del pavimento utilizando un haz de luz artificial para operar por la noche. Con la implementación de este sistema se extrae información de la falla sobre la película a través de una mesa especial para su despliegue. Este equipo se utilizó ampliamente en países como Francia, España, Portugal y Túnez (Hudson et al., 1987). Earth Technology Corporation fue otra de las compañías que utilizó cámaras de escaneo para adquirir datos de daños que eran posteriormente analizados para evaluar la condición del pavimento, su sistema contaba con cuatro cámaras de 512 píxeles de resolución para realizar una medida de $2.43 \mathrm{~m}$ de ancho (Díaz, 2013).

Roadware Corporation desarrolló el analizador automático de caminos (ARAN), el cual entrega medidas de la profundidad de rodadura por medio de siete sensores ultrasónicos, instalados en la sección frontal del vehículo. También utiliza una cámara de video instalada en el parabrisas para grabar el lado derecho del camino y otra para tomar un video de la superficie del pavimento, usando un operador que dispone de dos teclados para grabar los datos de las fallas, el nivel de severidad y el nivel de magnitud (Hudson et al., 1987).

El sistema láser RST (Road Surface Tester), desarrollado en Suecia, utiliza tecnología láser para medir la profundidad de las grietas, su anchura y la profundidad de la rodadura de neumático, es capaz de medir la distancia recorrida, el perfil longitudinal y la macrotextura de la superficie. Un ordenador integra los datos de campo capturados para distintas secciones de vía analizadas proporcionando información en tiempo real (Solminihac, 2001).

Entre otros sistemas, que utilizan captación de imágenes para procesos de gestión de pavimentos, se encuentra el RoadCrack, de la compañía australiana CSIRO, que incluye cámaras y herramientas de procesamiento de imágenes para detectar y clasificar fisuras del pavimento. El sistema VCrack del Departamento de Transporte de Texas, que consiste en una cámara de escaneo por línea con sensor tipo CCD (Dispositivo acoplado por carga), una tarjeta de captación de imágenes y una computadora que es capaz de caracterizar las grietas en tiempo real. El sistema Crackscope, a cargo del Centro de Investigación en Transporte de la Universidad de Texas en Austin, utiliza una cámara de escaneo por línea con una resolución de 2,048 píxeles, interfaz de comunica- ción Gigabit Ethernet y un proyector láser para hacer capturas de hasta $3.6 \mathrm{~m}$ de ancho de carril sobre la vía.

En el ámbito nacional colombiano, el sistema SICAIPAV, desarrollado por investigadores de la Universidad Industrial del Santander, captura imágenes de fallas del pavimento a través de una cámara de escaneo por línea, con resolución de 4,096 píxeles, instalada en un vehículo de inspección, que logra hacer registros de hasta $4 \mathrm{~m}$ de ancho con una precisión de $1 \mathrm{~mm} /$ píxel. Este sistema mejoró aplicando características geométricas y descriptores de forma (Díaz, 2012, 2013, 2014). Investigaciones realizadas en esta materia por Wang (2000) indicaron que los dispositivos basados en cámaras de escaneo por línea tienen mayores ventajas para el registro de deterioros superficiales en los pavimentos que los obtenidos con implementación de dispositivos láser.

\section{El sistema propuesto}

El sistema propuesto permite generar mapas de intervención vial que detallan, a nivel de red, la ubicación de daños severos existentes sobre el pavimento y sus características. Hacer esto en forma objetiva implica estandarizar los procesos de medición, tales como la localización de los daños, de tal manera que sea posible hacer análisis espaciales con ayuda de software especializado como ARCGIS (Zhou et al., 2009; Verhoef et al., 2001; McCoy, 2004; Shrestha y Shrestha, 2014).

La consecuencia inmediata es el fortalecimiento del criterio visual que emplean los administradores viales para la valoración del estado de las redes de transporte, reduciendo así diagnósticos subjetivos y apoyando la toma de decisiones en la formulación de mejores políticas de inversión vial, como se ilustra en la figura 1.

Para recopilar de forma rápida, precisa y automática la información de campo se propone la fabricación de un dispositivo electrónico compuesto por los siguientes elementos: una placa microcontroladora basa-
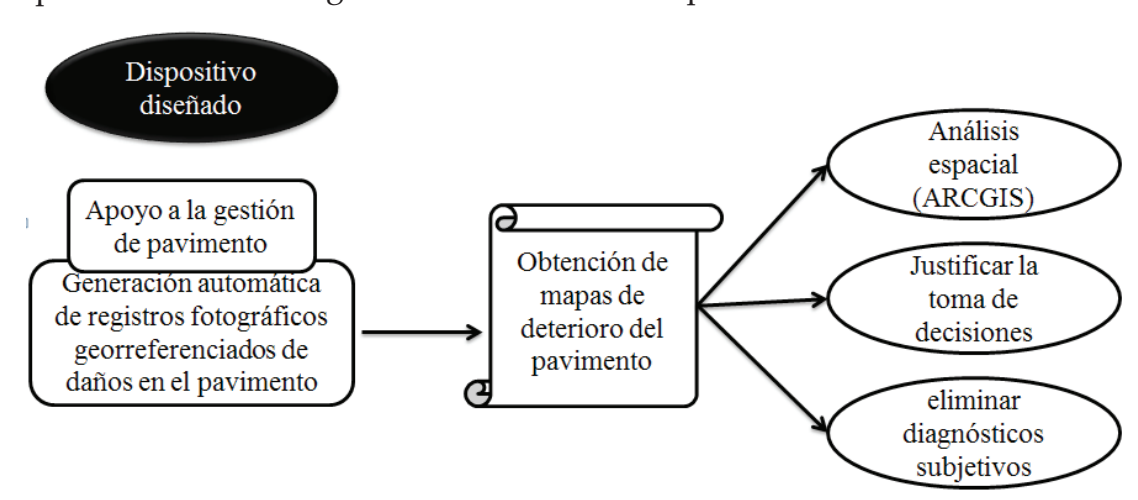

Figura 1. Alcances del dispositivo de inventariado de daños 


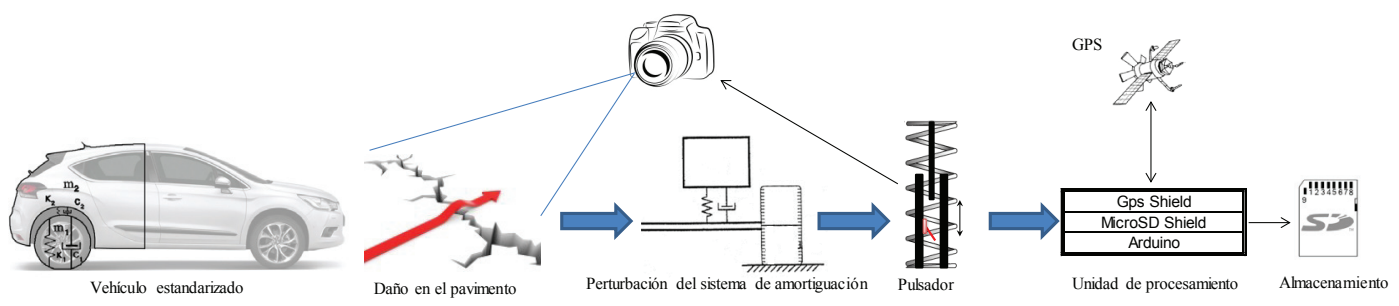

Figura 2. Procesos del sistema de inventariado de daños propuesto

da en un procesador ATmega328 (Arduino uno), una placa GPS shield de arduino, una tarjeta microSD shield, una cámara digital y un mecanismo de activación por perturbación (MAPP) adaptado al sistema de amortiguación de un vehículo.

La interacción conjunta de estos elementos permitirá la detección, captura y georreferenciación automática de los daños existentes sobre la red vial, en respuesta a las perturbaciones percibidas en el sistema de amortiguación de un vehículo de inspección, al pasar por diversos tipos de deterioro y ondulaciones existentes en la superficie del pavimento, aspecto que se relaciona directamente con el grado de confort que experimentan los usuarios de las vías, y con la funcionalidad y servicio que estas prestan. La figura 2 muestra un esquema del sistema propuesto para el inventariado de daños.

La arquitectura completa del sistema se ilustra en la figura 3, que muestra la interacción entre los principales componentes del sistema hardware para obtener la información de campo requerida. Esta información se extrae y administra a través un sistema de administración de datos (SAD).

\section{Dispositivo de inventariado de daños en el pavimento}

El proceso de detección de daños del pavimento utiliza un mecanismo de activación por perturbación, que consiste en la ubicación de un sensor o pulsador ${ }^{1}$ dentro del sistema de amortiguación de un vehículo de inspección, calibrado para accionarse al nivel de perturbación deseado. Este mecanismo se encarga de enviar señales cada vez que detecta alguna de las tipologías de daño estructural o funcional que hacen parte de los alcances del dispositivo, es decir, baches, agrietamientos de gran magnitud, escalonamiento y levantamiento pronunciado de juntas en pavimentos rígidos, ahuellamientos y hundimientos severos y ondulaciones tanto transversales como por desplazamiento en pavimentos asfálticos. El dispositivo no identifica deterioros superficiales.

1 Un pulsador es un operador eléctrico que, cuando se oprime, permite el paso de la corriente eléctrica $y$, cuando se deja de oprimir, lo interrumpe.
La señal generada por el MAPP activa el disparo de una cámara digital con capacidad de estabilización de imagen, previamente instalada en la parte posterior del vehículo utilizado y calibrada según la velocidad de circulación. La señal acciona el protocolo de georreferenciación, así como el proceso de seriado, almacenamiento y asociación de la información capturada, mediante la interacción del proceso de programación con los componentes: Arduino Uno, GPS Shield y MicroSD Shield. La tabla 1 detalla algunas características de los principales componentes del dispositivo propuesto.

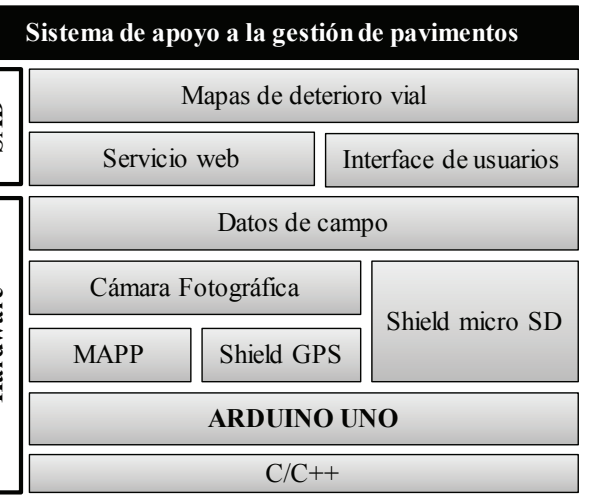

Figura 3. Arquitectura del sistema diseñado

Tabla 1. Características de los principales componentes del dispositivo de inventariado

\begin{tabular}{ll}
\hline \multicolumn{1}{c}{ Componente } & \multicolumn{1}{c}{ Características } \\
\hline $\begin{array}{l}\text { Componente lógico } \\
\text { de procesado }\end{array}$ & $\begin{array}{l}\text { Arduino Uno R3 con placa electrónica } \\
\text { ATmega328, encargado de controlar la } \\
\text { lógica de programación del dispositivo } \\
\text { en lenguaje C++ } \\
\text { Shield GPS y receptor GPS EM406 con } \\
\text { regulación de voltaje, indicador de } \\
\text { estado LED, batería de respaldo RAM, y } \\
\text { antena de parche. Esta componente emite } \\
\text { continuamente señales a los satélites } \\
\text { presentes en la zona, permitiendo } \\
\text { georreferenciación } \\
\text { georreferenciar la ubicación de los puntos } \\
\text { asociados a los daños } \\
\text { Utiliza una Shield MicroSD para } \\
\text { almacenar los datos de campo: latitud y } \\
\text { longitud del punto evaluado, fotografía } \\
\text { de los daños y código de asociación }\end{array}$ \\
\hline
\end{tabular}


La interconexión electrónica de los componentes del sistema se detalla en la figura 4. La asociación de estos componentes con una cámara digital permite conformar la unidad principal de procesamiento de datos del sistema (figura 5), que se encarga de capturar y almacenar la información de campo destinada a fortalecer los procesos de gestión de pavimentos. Se busca que la información sea la estrictamente requerida para facilitar la apropiada toma de decisiones a nivel estratégico, evitando comprometer la eficacia del sistema de gestión con detalles excesivos que incrementarían los costos de procesamiento al requerir equipos sofisticados (Bennett et al., 2006).

La unidad de procesamiento de datos se instala en la parte posterior del vehículo y se interconecta con el MAPP, que se gradúa en función de la velocidad de recorrido y la masa del vehículo. Los datos obtenidos se almacenan en la microSD Shield y se pueden analizar en oficina a través del sistema de administración de datos (SAD), que genera mapas de deterioro a nivel de red. Además, los datos se procesan en cualquier plataforma de análisis de información geográfica, proporcionando un registro permanente de la condición de la superficie vial, lo que reduce los costos de recolección de información de campo para los sistemas de gestión de pavimentos.

Una ventaja del dispositivo propuesto, en términos de capacidad de almacenamiento, es que solo se enfoca en daños que afectan el índice de servicio y confort de los usuarios y no en daños superficiales poco perceptibles, cuyo registro aumentaría el consumo de memoria del dispositivo. No obstante, la capacidad de almacenamiento del sistema está restringida por la captura fotográfica a un máximo de 15,750 registros, tal como se aprecia en la tabla 2.

El dispositivo requiere \pm 1 segundo para enfocar la cámara fotográfica $\mathrm{y} \pm 1$ segundo para la secuencia de disparo, detección de coordenadas y almacenamiento de la información. En consecuencia, la latencia del sistema es de aproximadamente 2 segundos/registro, lo que permite hacer un único registro para eventos generados consecutivamente en un lapso inferior a este tiempo.

En cuanto al mecanismo de activación por perturbación, este se puede configurar para ser sensible ante diferentes severidades de da- ños y ondulaciones sobre el pavimento. Para esto se requiere calibrar la distancia de activación $\Delta x$ (figura 6), que separa la barra de movimiento del sensor o pulsador, considerando tanto la masa del vehículo como la velocidad de operación.

En definitiva se pueden destacar las siguientes características del dispositivo:

1. Sencillez. Lo que a su vez proporciona un mejor y más rápido funcionamiento e interconectividad con todos los elementos del sistema.

2. Potabilidad. Es un dispositivo de fácil instalación y desmonte.

3. Usabilidad. El dispositivo se puede adaptar a diferentes tipos de vehículo.

4. Sensibilidad. La generación de señales de activación son ajustables a la magnitud de los daños y a la percepción de los usuarios.

5. Facilidad de uso. El encendido, operación y extracción de los datos es simple, a través de microSD.

6. Compatibilidad. El formato de la información almacenada es compatible con los sistemas de información geográfica más utilizados.

7. Economía. Se prevé que los costos, tiempo y logística para inventariado de daños sean inferiores a los incurridos a través de métodos manuales como el PCI.
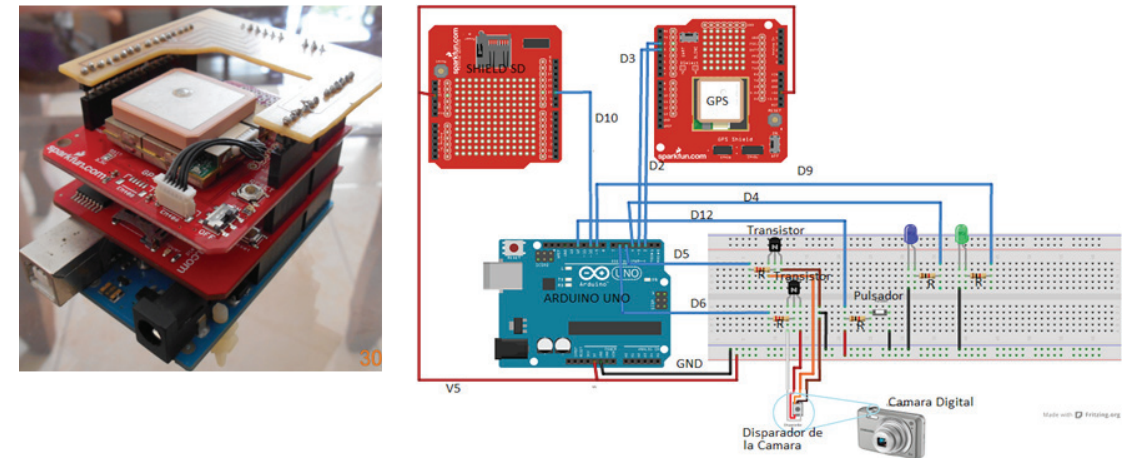

Figura 4. Descripción e interconexión de los componentes del dispositivo propuesto D: Conexión digital; V: Voltaje; GND: Conexión a Tierra; R: Resistencia eléctrica
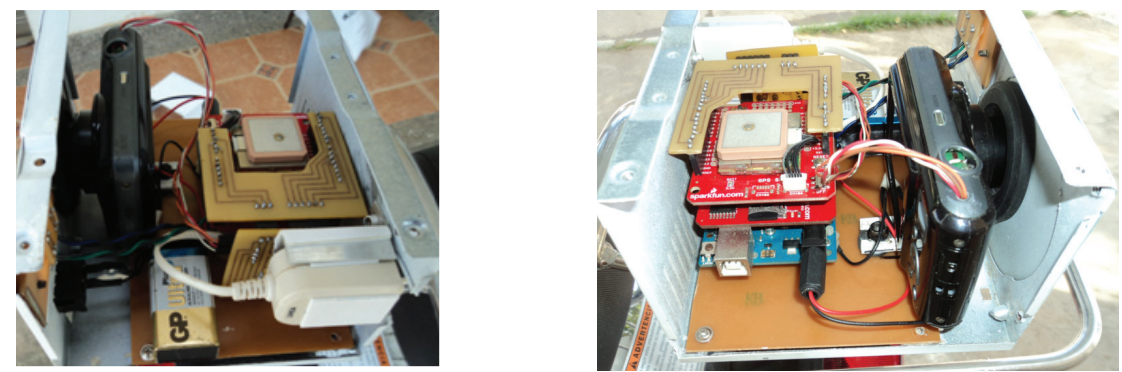

Figura 5. Unidad principal de procesamiento de datos 
Tabla 2. Capacidad del sistema de almacenamiento

\begin{tabular}{ccc}
\hline Descripción & $\begin{array}{c}\text { Coordenadas geográficas y } \\
\text { código de asociación }\end{array}$ & Captura fotográfica \\
\hline Tamaño en memoria por evento & $\approx 40$ bytes/evento & $\approx 2.0$ MB/evento \\
Tamaño de tarjetas SD soportado & $2 \mathrm{~GB}$ & $32 \mathrm{~GB}$ \\
Capacidad total por registro & $\approx 53^{\prime} 687,091$ & $\approx 15,750$ \\
\hline
\end{tabular}

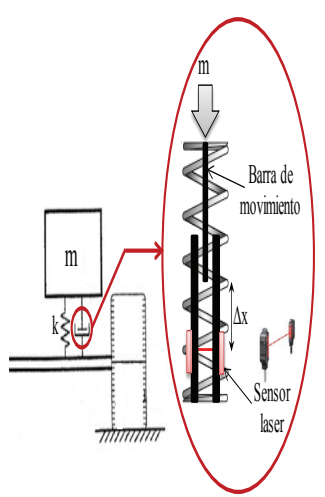

a)

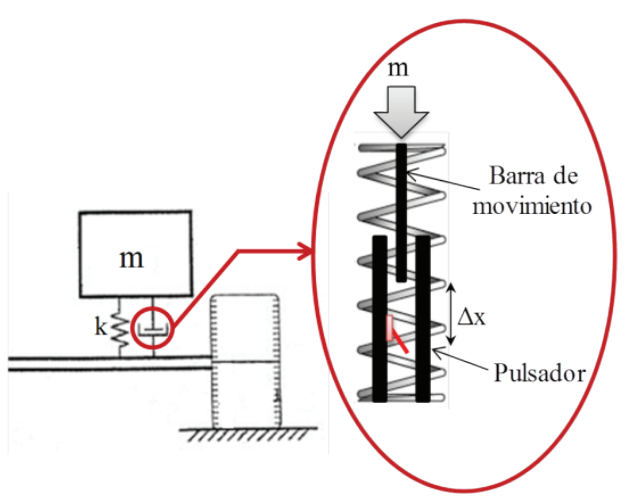

b)

Figura 6. Mecanismo de activación por perturbación (MAPP), a) basado en el uso del sensor, b) basado en el uso del pulsador

\section{Sistema de administración de datos (SAD)}

El sistema de administración de datos es una extensión del dispositivo desarrollado, que es parte integral del sistema mediante plataformas WEB, transforma los datos en mapas que contienen el recorrido del vehículo, la ubicación geográfica de los deterioros y el detalle fotográfico de los daños capturados en el pavimento. Este tipo de información le otorga a los tomadores de decisión la capacidad de programar con eficiencia diversos tipos de tratamientos. La figura 7 ilustra los alcances y la arquitectura del SAD.

La pestaña "subir archivos" de la interfaz del administrador del sitio WEB (figura 8), permite cargar tanto las imágenes como el archivo .csv generado por el dispositivo electrónico después de su recorrido por la red vial evaluada. Adicionalmente es posible editar algunos atributos de la vía, tales como: ubicación políticoadministrativa del tramo evaluado, código de la vía, nombre, kilómetro inicial y final del recorrido, número de calzadas, carriles por calzada y ancho de los carriles, entre otros, tal como lo propone la dirección nacional de vialidad de Chile (Solminihac, 2001).

La pestaña "Historial" del sitio WEB permite acceso a la base de datos de los tramos evaluados en diferentes fechas, visualizando la información general de la ruta y el mapa de deterioros con su respectivo registro fotográfico, brindando la posibilidad de descarga en formato $\mathrm{kml}$. La figura 9 muestra en vista previa la información de dos recorridos, cuyo mapa de daños puede acceder directamente a través de Google Maps utilizando el botón "ver KML en google maps", dando así una idea de la condición en la que se encuentran las rutas.

Utilizando "Editar Reporte" se pueden crear reportes con la patología de los daños encontrados en cada uno de los recorridos almacenados dentro de la base de datos del sistema. La figura 10 muestra la interfaz para la edición de patologías. Esta ventana le brinda al administrador la posibilidad de definir para cada daño su tipología, severidad, carril de ubicación, comentarios y la imagen que representa el daño evaluado. De esta forma, es posible reducir tiempo y costo en la adquisición,

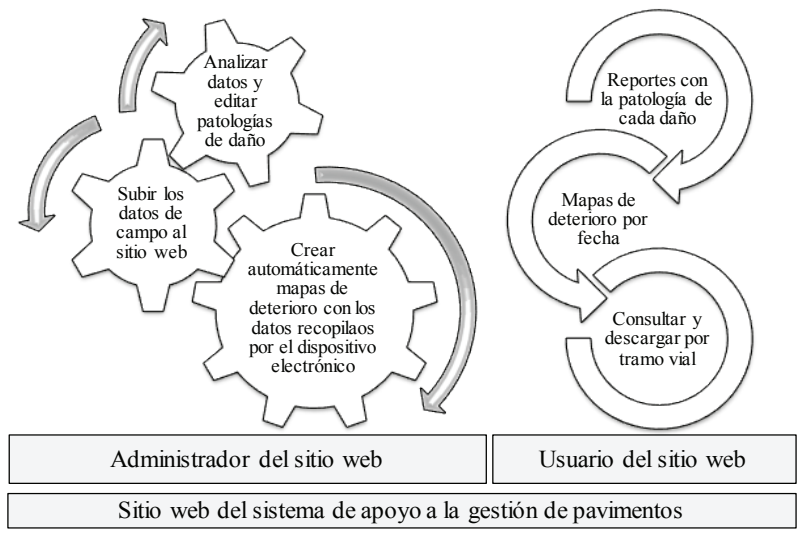

Figura 7. Arquitectura del sistema de administración de datos-SAD 


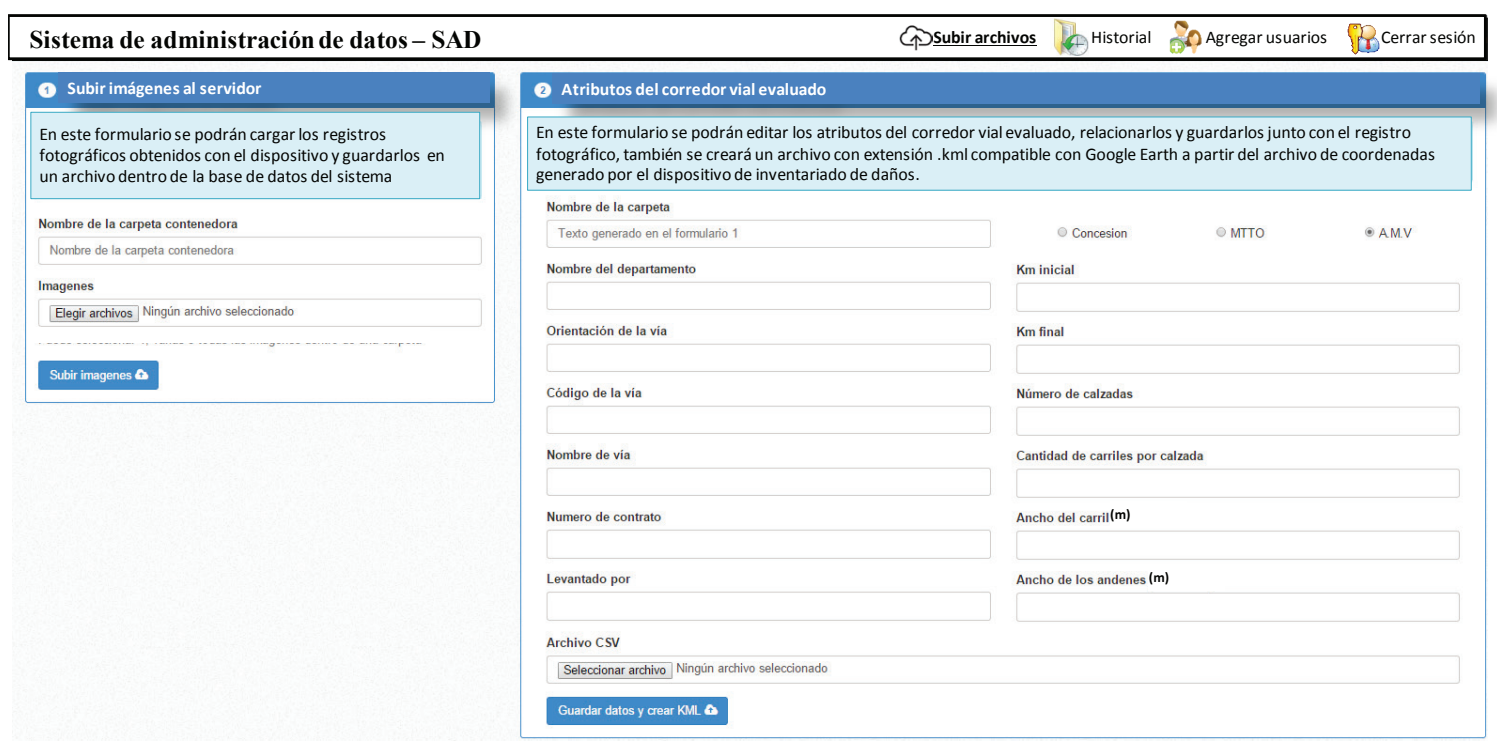

Figura 8. Interfaz para el cargue de datos de campo

Sistema de administración de datos - SAD

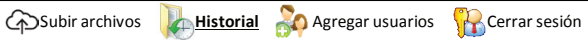

\begin{tabular}{|c|c|c|c|c|}
\hline \multicolumn{5}{|c|}{ Datos de la vía } \\
\hline Departamento: & Córdoba & Km inicial: & 15 & Vor KML on Google Maps \\
\hline Orientación: & Sincelejo - Montería & Km final: & 117 & \\
\hline Código de la vía: & Rutas 25 y 74 & Número de calzzdas: & 1 & ^ Descargar KM \\
\hline Nombre de la vía: & Troncal de occidente & Carriles por calzada: & 2 & \\
\hline Fecha de registro: & Lunes Nov 102014 & Ancho de los carriles: & $3.0 \mathrm{~m}$ & 를 Editar Reporte \\
\hline Nümero de contrato: & 00001 & Ancho de los andenes: & $1 \mathrm{~m}$ & \\
\hline \multicolumn{5}{|c|}{ Datos de la vía } \\
\hline Departamento: & Nariño & Km inicial: & 2 & Ver KML en Google Maps \\
\hline Orientación: & Mojarras - Rumichaca & Km final: & 209.6 & \\
\hline Código de la via: & Ruta 25 & Número de calzadas: & 1 & \& Descargar KM \\
\hline Nombre de la via: & Tramo 2501 & Carriles por calzada: & 2 & \\
\hline Fecha de registro: & Miércoles Enero 142015 & Ancho do los carrilos: & $3.5 \mathrm{~m}$ & EEditar Reporte \\
\hline Número de contrato: & 00002 & Ancho de los andenes: & $1 \mathrm{~m}$ & \\
\hline
\end{tabular}

Figura 9. Interfaz con información de dos recorridos

Sistema de administración de datos - SAD G Gubir archivos

\begin{tabular}{|l|l|l|l|l|l|}
\hline Patologias & \multicolumn{2}{|c|}{ Cocrdenadas (lat,log) } & Aclaciones & Nombre Imagen \\
\hline Carril & Tipo de daño & Severidad & Cubre todo el ancho de calzada & 2 \\
\hline E & PC & A & $8.971093,75.454032$ & & 3 \\
\hline D & FL, FT & A & $8.971093,75.454032$ & Presencia de suelo blando & 4 \\
\hline C & HUN & A & $8.1234567,-75.12345$ & Se evidencia desprendimiento & 8 \\
\hline D & FL, FT & A & $8.934867^{\circ},-75.442558^{\circ}$ & & 12 \\
\hline D & FL, FT & A & $8.908921^{\circ}, 75.447441^{\circ}$ & Grieta cubierta anteriormente & 14 \\
\hline D & FL, FT & A & $8.904012^{\circ}, 75.452896^{\circ}$ & \\
\hline
\end{tabular}

Agregar patologia

\begin{tabular}{|c|c|c|c|c|c|c|}
\hline Carril & I | Carril Izquierdo & $\mathbf{v}$ & Lat & Long & & Aclaraciones \\
\hline Tipo de daño & FL, FT | Fisuras longitudir & $\boldsymbol{v}$ & Severidad & Alta & $\boldsymbol{v}$ & Imágen \\
\hline
\end{tabular}

Figura 10. Interfaz para la edición de patologías por daño 
organización y posterior análisis de la información de campo, así como hacer una administración vial a nivel de red con adecuados procesos de planificación y programación.

\section{Caso de aplicación}

Gracias a los bajos costos de operación, se utilizó una motocicleta para validar la funcionalidad del dispositivo. El mecanismo de activación por perturbación se construyó siguiendo el prototipo de la figura $6 \mathrm{~b}$, activando la señal mediante un pulsador instalado en el sistema de amortiguación. La figura 11 muestra el MAPP construido, que se adaptó al sistema de amortiguación delantera de la motocicleta (figura 12), calibrando la distancia de activación $\Delta x$, de acuerdo con la masa del vehículo más la masa del conductor y la velocidad de operación establecida.

La unidad de procesamiento de datos se instaló en la parte posterior de la motocicleta (figura 13). El ángulo de la cámara se fijó internamente para capturar los daños sobre el pavimento en panorámica, en función de su velocidad.

Una vez interconectados ambos componentes se realizó un recorrido de $8.56 \mathrm{~km}$ por la vía troncal de occidente que cruza al municipio de Sahagún, en el departamento de Córdoba, Colombia. Durante el recorrido se registraron 16 irregularidades en la superficie del pavimento (figura 14a), que corresponden a resaltos y

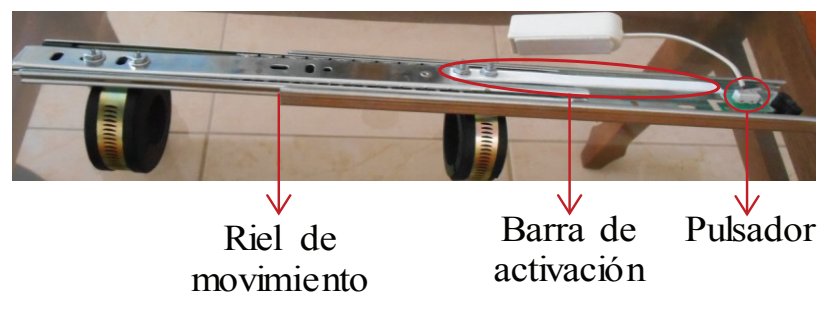

reductores de velocidad localizados por las autoridades de tránsito y transporte. Estas irregularidades incrementan los costos operacionales sobre los corredores viales, reducen la velocidad de operación del tráfico por los daños que pueden ocasionar en los vehículos, y a altas velocidades inducen pérdidas de confort en los usuarios de la vía.

La visualización en el mapa del detalle de los daños capturados por el dispositivo es posible a través del vínculo que asocia cada punto con el registro fotográfico de los daños (figura 14b), el cual se despliega por defecto al acercar el puntero.

Figura 11. Mecanismo de activación por pulsador propuesto

\section{Confiabilidad del dispositivo}

Para validar la confiabilidad del dispositivo se tomaron dos muestras de coordenadas geográficas en 2 puntos ubicados sobre una sección de vía en línea recta, sobre la cual se colocaron pequeños resaltos para simular daños puntuales sobre el pavimento. La primera muestra se tomó utilizando un GPS convencional ubicado exactamente sobre cada uno de los puntos indicados. En la tabla 3 se describen las características de esta muestra.

Posteriormente se hizo circular el vehículo 15 veces sobre la sección de vía en consideración a una velocidad constante de $30 \mathrm{~km} / \mathrm{h}$, obteniendo un total de 15 lecturas de latitud y longitud sobre cada punto. La media, la desviación estándar y otras medidas de interés

de la muestra obtenida se presentan en la tabla 4.
\end{abstract}

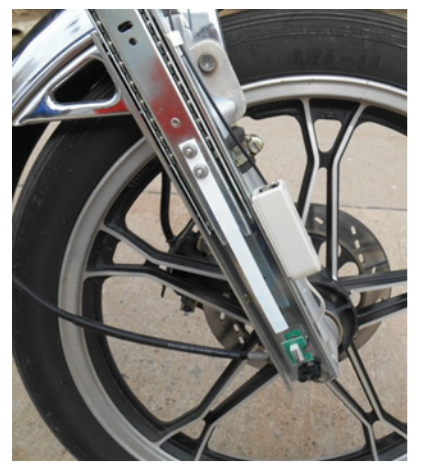

Figura 12. Adaptación del mecanismo de activación por perturbación construido

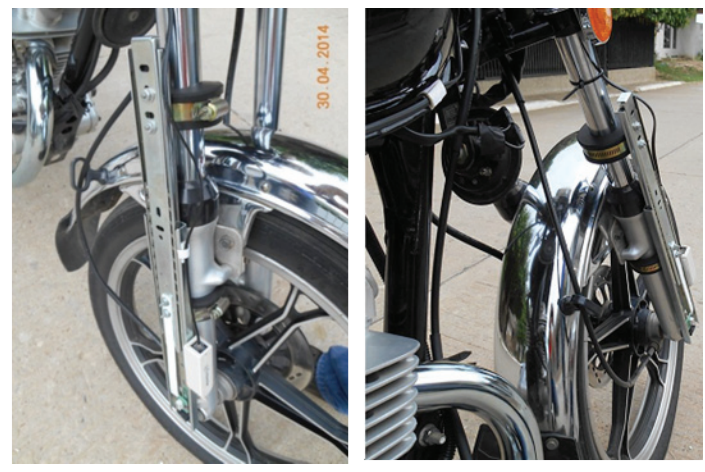

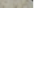

(n)




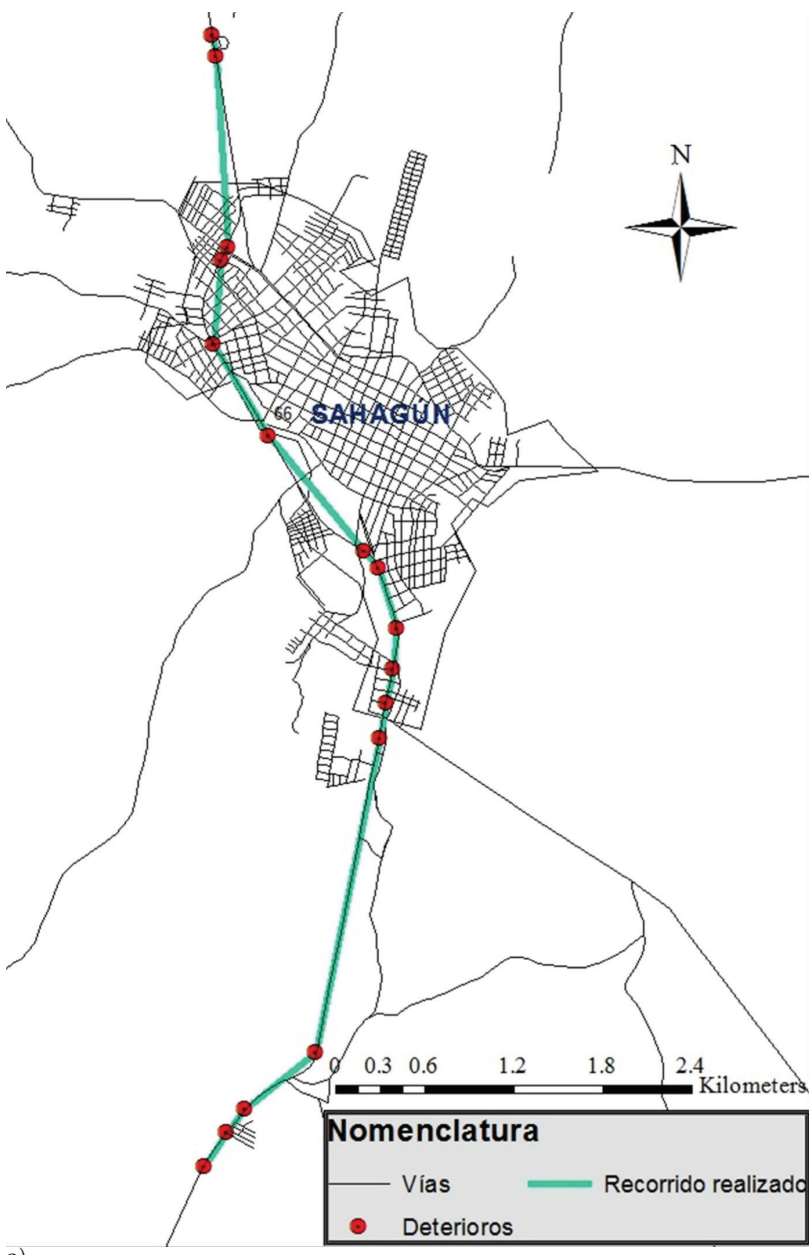

a)

Figura 14. Mapa de deterioro obtenido, a) mapa con daños capturados, b)

Se tienen solamente algunas observaciones en cada caso (menos de 30), así que el teorema del límite central no se aplica. Al suponer que ambas poblaciones siguen distribuciones normales, la distribución t-Student se puede utilizar para construir una prueba de hipótesis, con la cual se pueda verificar la factibilidad de que las medias poblacionales de ambas muestras son iguales y de que la diferencia entre las medias muestrales es atribuible solamente a la variación aleatoria. Los resultados obtenidos en esta prueba de hipótesis se muestran en la tabla 5.

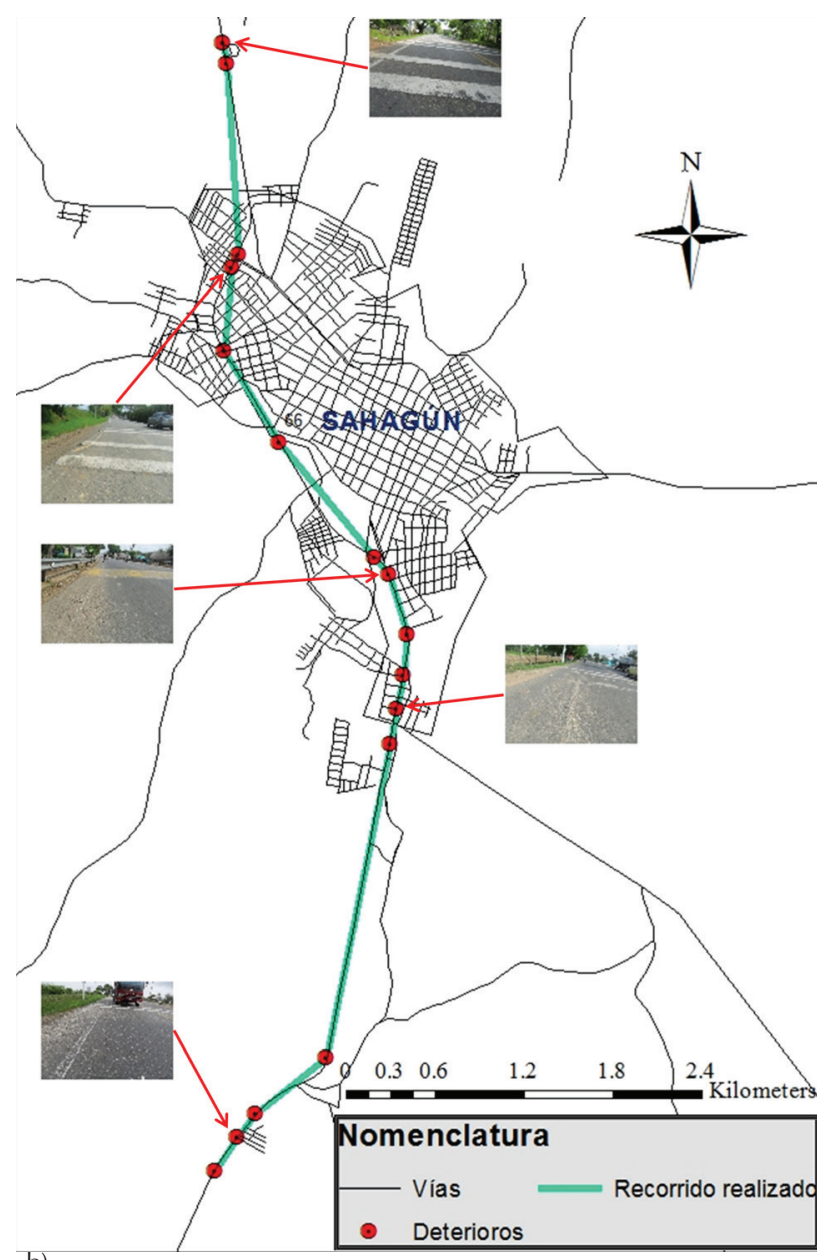

Esta es una prueba de dos colas, y el p-valor en todos los casos fue muy grande, por tanto se puede establecer en forma concluyente, que es razonable ser optimista en cuanto a la veracidad de la hipótesis de que la media de las coordenadas obtenidas a través del vehículo de inspección equipado con el dispositivo es muy cercana a la media obtenida con un GPS ubicado exactamente sobre los puntos que se evalúan.

Tabla 3. Muestra de coordenadas obtenida con GPS convencional (sin vehículo)

\begin{tabular}{lcccc}
\hline \multirow{2}{*}{\multicolumn{1}{c}{ Indicadores estadísticos }} & \multicolumn{2}{c}{ Punto 1 } & Latitud & Longitud \\
\cline { 2 - 5 } & Latitud & Longitud & 16 & 16 \\
\hline Tamaño muestral & 16 & 16 & 8.95116425 & -75.443666 \\
Media muestral & 8.95104563 & -75.443724 & $1.8697 \mathrm{E}-06$ & $2.4595 \mathrm{E}-06$ \\
Error típico & $2.3855 \mathrm{E}-06$ & $4.1922 \mathrm{E}-06$ & $7.4789 \mathrm{E}-06$ & $9.8379 \mathrm{E}-06$ \\
Desviación estándar & $9.5420 \mathrm{E}-06$ & $1.6769 \mathrm{E}-05$ & $2.1 \mathrm{E}-05$ & $3 \mathrm{E}-05$ \\
Rango & $3.8 \mathrm{E}-05$ & $6.1 \mathrm{E}-05$ & 8.951152 & -75.443679 \\
Mínimo & 8.951025 & -75.443771 & -75.951173 & -75.443649 \\
Máximo & 8.951063 & -75.44371 & 8.953 \\
\hline
\end{tabular}


Tabla 4. Muestra de coordenadas obtenida con el vehículo equipado con el dispositivo

\begin{tabular}{lcccc}
\hline \multirow{2}{*}{\multicolumn{1}{c}{ Indicadores estadísticos }} & \multicolumn{2}{c}{ Punto 1 } & Punto 2 \\
\cline { 2 - 5 } & Latitud & Longitud & Latitud & Longitud \\
\hline Tamaño muestral & 15 & 15 & 15 & 15 \\
Media muestral & 8.95104673 & -75.443728 & 8.95116353 & -75.443662 \\
Error típico & $1.7821 \mathrm{E}-06$ & $6.4779 \mathrm{E}-06$ & $2.4238 \mathrm{E}-06$ & $2.3234 \mathrm{E}-06$ \\
Desviación estándar & $6.9020 \mathrm{E}-06$ & $2.5089 \mathrm{E}-05$ & $9.3874 \mathrm{E}-06$ & $8.9984 \mathrm{E}-06$ \\
Rango & $2.4 \mathrm{E}-05$ & $6.1 \mathrm{E}-05$ & $2.1 \mathrm{E}-05$ & $2.3 \mathrm{E}-05$ \\
Mínimo & 8.951039 & -75.44377 & 8.951152 & -75.443672 \\
Máximo & 8.951063 & -75.44371 & 8.951173 & -75.443649 \\
\hline
\end{tabular}

Tabla 5. Resultados de la prueba de hipótesis

\begin{tabular}{|c|c|c|c|c|}
\hline \multirow{2}{*}{ Parámetros estadísticos } & \multicolumn{2}{|c|}{ Punto 1} & \multicolumn{2}{|c|}{ Punto 2} \\
\hline & Latitud & Longitud & Latitud & Longitud \\
\hline $\bar{X}-\bar{Y}$ & 1.10833E-06 & $-4.637 \mathrm{E}-06$ & $-7.167 \mathrm{E}-07$ & $4.775 \mathrm{E}-06$ \\
\hline Desviación estándar conjunta & 2.97767E-06 & 7.7161E-06 & $3.0612 \mathrm{E}-06$ & 3.3833E-06 \\
\hline Grados de libertad $(v)$ & 27 & 24 & 26 & 28 \\
\hline Estadístico t-student $(t)$ & 0.372215398 & 0.6010143 & -0.2341154 & 1.41132332 \\
\hline p-valor $(1$ cola $)$ & 0.356319054 & 0.2767325 & 0.40836457 & 0.08458259 \\
\hline p-valor $(1$ cola $)$ & 0.712638107 & 0.5534650 & 0.81672914 & 0.16916518 \\
\hline
\end{tabular}

\section{Conclusiones}

Se propuso un sistema de apoyo a la gestión de pavimentos basado en el uso de nuevas tecnologías y sistemas de información geográfica, que facilita la captura y análisis de la información de campo requerida para los procesos de administración vial. El sistema planteado utiliza un vehículo equipado con dispositivos electrónicos de bajo costo que permiten el registro y localización geográfica automática de daños existentes en los pavimentos, así como la generación de mapas de deterioro vial que se pueden acceder a través de plataformas WEB. La gestión de esta información de campo posibilita justificar acertadamente la toma de decisiones en torno a políticas de inversión en infraestructura vial, ahorro de costos de operación en el registro de la información de campo y la eliminación de diagnósticos subjetivos de evaluación.

La implementación del sistema implicó el diseño de un dispositivo electrónico para la detección automática de daños del pavimento, el cual captura información georreferenciada que se puede utilizar a través de cualquier plataforma de análisis de información geográfica. Esta característica garantiza la compatibilidad de los datos con la mayoría de las plataformas informáticas disponibles en las entidades responsables de la gestión de infraestructura y seguridad vial. Como una extensión del dispositivo para el inventariado de daños se propuso un sistema de administración de datos, el cual permite, a través de plataformas WEB, transformar los datos de campo obtenidos con el dispositivo en mapas que contienen el recorrido hecho por el vehículo, la ubicación geográfica de los deterioros y el detalle fotográfico de los daños capturados en el pavimento.
El dispositivo para el inventariado de daños quedó finalmente conformado por los siguientes componentes: una placa microcontroladora basada en un procesador ATmega328 (Arduino uno), una placa GPS shield de arduino, una tarjeta microSD shield, una cámara fotográfica y un mecanismo de activación por perturbación (MAPP) adaptado al sistema de amortiguación de un vehículo. Dicho sistema es portable, de fácil instalación y manejo, confiable y eficiente a la hora de obtener información de campo a bajo costo.

Las pruebas realizadas para validar la confiabilidad del dispositivo son optimistas en cuanto a la veracidad de la hipótesis nula de que la media de las coordenadas obtenidas a través del vehículo de inspección equipado con el dispositivo es muy cercana a la media obtenida con un GPS ubicado exactamente sobre los puntos que se evalúan.

Finalmente, es importante advertir que el criterio adoptado por el conductor del vehículo podría incidir en la correcta detección de los daños del pavimento. Esto es así porque el sistema depende de la reacción de la suspensión de la rueda del vehículo, cuya lectura se limita al ancho de la llanta. Entonces, sería necesario que el conductor dirigiera la rueda del vehículo hacia el punto defectuoso del pavimento, lo que representa un aspecto por mejorar.

\section{Agradecimientos}

Los autores expresan sus agradecimientos al Departamento de Ingeniería de Sistemas de la Universidad de Córdoba, Montería, Colombia, así como al Banco Santander y UNIVERSIA Colombia, a través del programa de Beca Iberoamérica, Jóvenes Profesores e Investiga- 
dores, Santander Universidades 2015, por su apoyo técnico y financiero.

\section{Referencias}

Bennett C.R., De-Solminihac H., Chamorro A. Data collection technologies for road management, Transport Note No. 30: 1-8, The World Bank, Washington, DC, 2006.

Bull A. Un nuevo paradigma para la conservación vial: de hacer lo que se puede a hacer lo que es exigible, División de Recursos Naturales e Infraestructura, CEPAL, Santiago, Chile, Primer congreso de fondos de conservación vial de Centroamérica, 2003.

Corporación fondo de prevención vial de Colombia, Quiénes somos, 2013 [en línea] [fecha de consulta: 8 de febrero de 2013]. Disponible en: www.fpv.org.co/

Díaz H.P., Echeverry D.Y.S., Melgarejo Y.H.M. Sistema automático para la adquisición de imágenes de vías pavimentadas. Revista Gerencia Tecnología Informática, volumen 12 (número 32), 2013: 61-78.

Díaz H.P., Pinzón E.A.C., Echeverry D.Y.S., Pérez G.M.M. Detección automática de grietas de pavimento asfáltico aplicando características geométricas y descriptores de forma. INGE CUC, volumen 8 (número 1), 2012: 261-280.

Díaz H.P., Suárez J.H.R., Melgarejo Y.H.M., Rodríguez J.M.P. Sistemas automáticos para la adquisición de datos enfocados a examinar pavimentos flexibles. Ciencia e Ingeniería Neogranadina, volumen 24 (número 1), 2014: 79-98.

Echeverry-Arciniegas C.A. Evaluación de la metodología PCI como herramienta para la toma de decisiones en las intervenciones a realizar en los pavimentos flexibles, Universidad Militar Nueva Granada, Bogotá D.C., 2014 [en línea] [fecha de consulta: 30 de octubre de 2014]. Disponible en: http://repository.unimilitar.edu.co/bitstream/ 10654/12102/1.

Harwood D.W., Bauer K.M., Gilmore D.K., Souleyrette R., Hans Z.N. Validation of U.S. road assessment, program star rating protocol, application to safety management of U.S. roads. Journal of the Transportation Research Board, 2010: 33-41.

Hudson W.R., Elkins G.E., Uddin W., Reilley K.T. Improved methods and equipment to conduct pavement distress surveys, report No. FHWA-TS-87-213, Department of Transportation, Washington, DC, 1987.

INVIAS. Instituto Nacional de Vias, 2013 [en línea] [fecha de consulta: 16 de noviembre de 2014]. Disponible en: www.invias.gov.co

IRAP. International Road Assessment Programme [en línea] [fecha de consulta: 8 de febrero de 2013]. Disponible en: http:// www.irap.org./about-irap/about-us.

IRAP. Star Rating Roads For Safety: The iRAP Methodology, Hampshire, UK, International Road Assessment Programme (iRAP), 2009.

Kerali H.G.R. Overview of HDM-4. The Highway Development and Management Series, volumen 4, Association Mondiale de la Route AIPCR, 2000.
López-Candela C.R. Metodología para la recolección y análisis de información primaria como soporte al modelo de gestión de pavimentos en la red vial de Bucaramanga, Colombia, Facultad de ingenierías Fisicomecánicas, Universidad Industrial del Santander, 2010.

McCoy J. ArcGIS 9: Using ArcGIS Spatial Analyst, Esri Press, 2004. Miquel M.P., Henao J.P. Análisis de inversiones en carreteras utilizando Software HDM-4. Revista de la Construcción, volumen 6 (número 1), 2007: 35-47.

Shrestha K.K., Shrestha P.P. A GIS-enabled cost estimation tool for road upgrade and maintenance to assist road asset management systems, en: Construction Research Congress 2014@ sConstruction in a Global Network, ASCE, 2014, pp. 12391248.

Solminhac T., Hidalgo S., Salgado T., Valdés F. Calibración de modelos de comportamiento HDM de pavimentos asfálticos a las condiciones de Chile, Chile, estudio de seguimiento de pavimentos asfálticos, Ministerio de Obras Públicas, 2003.

Solminihac H. Gestión de infraestructura vial, Santiago de Chile, Ediciones Pontificia Universidad Católica de Chile, 2001, $508 \mathrm{p}$.

Thenoux G., Halles F. Metodología simplificada de priorización de conservación de pavimentos a nivel de red. Revista Ingeniería de Construcción, volumen 17 (número 1), 2011: 35-43.

Universidad de los Andes. A peinar las calles, 2013, [en línea] [fecha de consulta: 8 de febrero de 2013]. Disponible en http:// www.uniandes.edu.co/noticias/ingenieria/midiendo-calles

Verhoef J.M., Krivoruchko K., Lucas N. Using ArcGIS. Geostatistical analyst, volumen 380, Redlands, Esri, 2001.

Wang K.C. Designs and implementations of automated systems for pavement surface distress survey. Journal of Infrastructure Systems, volumen 6 (número 1), 2000: 24-32.

Zhou G., Wang L., Wang D., Reichle S. Integration of GIS and data mining technology to enhance the pavement management decision making. Journal of Transportation Engineering, volumen 136 (número 4), 2009: 332-341.

\section{Este artículo se cita:}

\section{Citación estilo Chicago}

Macea-Mercado, Luis Fernando, Luis Morales, Luis Gabriel Márquez-Díaz. Un sistema de gestión de pavimentos basado en nuevas tecnologías para países en vía de desarrollo. Ingeniería Investigación y Tecnología, XVII, 02 (2016): 223-235.

\section{Citación estilo ISO 690}

Macea-Mercado L.F., Morales L., Márquez-Díaz L.G. Un sistema de gestión de pavimentos basado en nuevas tecnologías para países en vía de desarrollo. Ingeniería Investigación y Tecnología, volumen XVII (número 2), abril-junio 2016:223-235. 


\section{Semblanzas de los autores}

Luis Fernando Macea-Mercado. Ingeniero civil por la Universidad de Cartagena (Cartagena D.T.C, Colombia), ingeniero de sistemas por la Universidad de Cordoba (Monteria, Colombia) y magister en ingenieria civil por la Universidad del Norte (Barranquilla, Colombia). Actualmente es candidato a doctor en ingenieria civil por la Universidad del Norte, donde ejerce como profesor e investigador en diversos proyectos de ingenieria civil relacionados con vias y transporte. Tambien se desempeña como profesor de vias en la Universidad de Sucre, Colombia y como consultor independiente en temas de movilidad.

Luis Morales. Ingeniero de sistemas por la Universidad de Córdoba, Colombia. Actualmente se desempeña en el área de las telecomunicaciones como contratista independiente. También se desempeña como desarrollador de aplicaciones Web.

Luis Gabriel Márquez-Díaz. Ingeniero de transporte y vias por la Universidad Pedagogica y Tecnologica de Colombia (UPTC). En 2008 obtuvo el grado de magister en ingeniería con énfasis en transporte en la UPTC y fue reconocido como el mejor estudiante de su cohorte. Es profesor asociado de la UPTC desde 2006. Ha participado como profesor en programas de posgrado en ingeniería de transporte en la Universidad Nacional de Medellin, Universidad del Norte, Universidad de Santander, Universidad Pontificia Bolivariana de Bucaramanga y en la Universidad Central del Ecuador. Actualmente es candidato a doctor en ingenieria civil por la Universidad del Norte, Colombia, donde adelanta su investigacion sobre modelacion híbrida en elecciones de transporte. 
DOI: https://doi.org/1 0.1016/j.riit.2016.06.007 